\title{
Association Between HLA Gene Polymorphism And The Genetic Susceptibility Of HIV Infection
}

\author{
Fang Yuan and Yongzhi Xi \\ Additional information is available at the end of the chapter \\ http://dx.doi.org/10.5772/57538
}

\section{Introduction}

Human leukocyte antigen (HLA) complex, which refers to a group of closely linked genes on the short arm of the sixth human chromosome, is considered the most polymorphic genetic marker that has so far been reported in the human. HLA plays a significant role in the immune response, particularly in antiviral immunity. Although HLA and genetic predisposition to various autoimmune diseases have been separately studied for the past 40 years, the evaluation of the correlation between the two was initiated only within the last 10 years. In recent years, research on HLA polymorphisms and susceptibility to various infectious diseases has attracted significant attention owing to the critical role of HLA in diseases such as SARS and hepatitis B. In particular, HLA polymorphism, HIV, and genetic predisposition to AIDS have emerged as research areas of immense clinical significance.

Human immunodeficiency virus (HIV) infection is able to perturb and alter gene expression through several mechanisms that can, lastly, cause acquired immunodeficiency syndrome (AIDS) Figure 1. Meanwhile, associations between disease parameters and the genetic makeup of the host and virus may be crucial in determining the outcome of HIV-1 infection.

According to Joint United Nations Programme on HIV/AIDS, there were approximately 40 million HIV-infected people worldwide at the end of 2004. Limiting the susceptibility to HIV, predicting the course of AIDS, and reversing it are some of the challenging tasks that need to be addressed urgently. Existing data demonstrates that the susceptibility to HIV differs among individuals, and significant differences exist in the disease progression in HIV-infected persons. In general, it takes less than 10 years from the time of HIV infection to the manifestation of typical AIDS symptoms. However, a small subset of HIV-infected people $(0.8 \%)$ are 


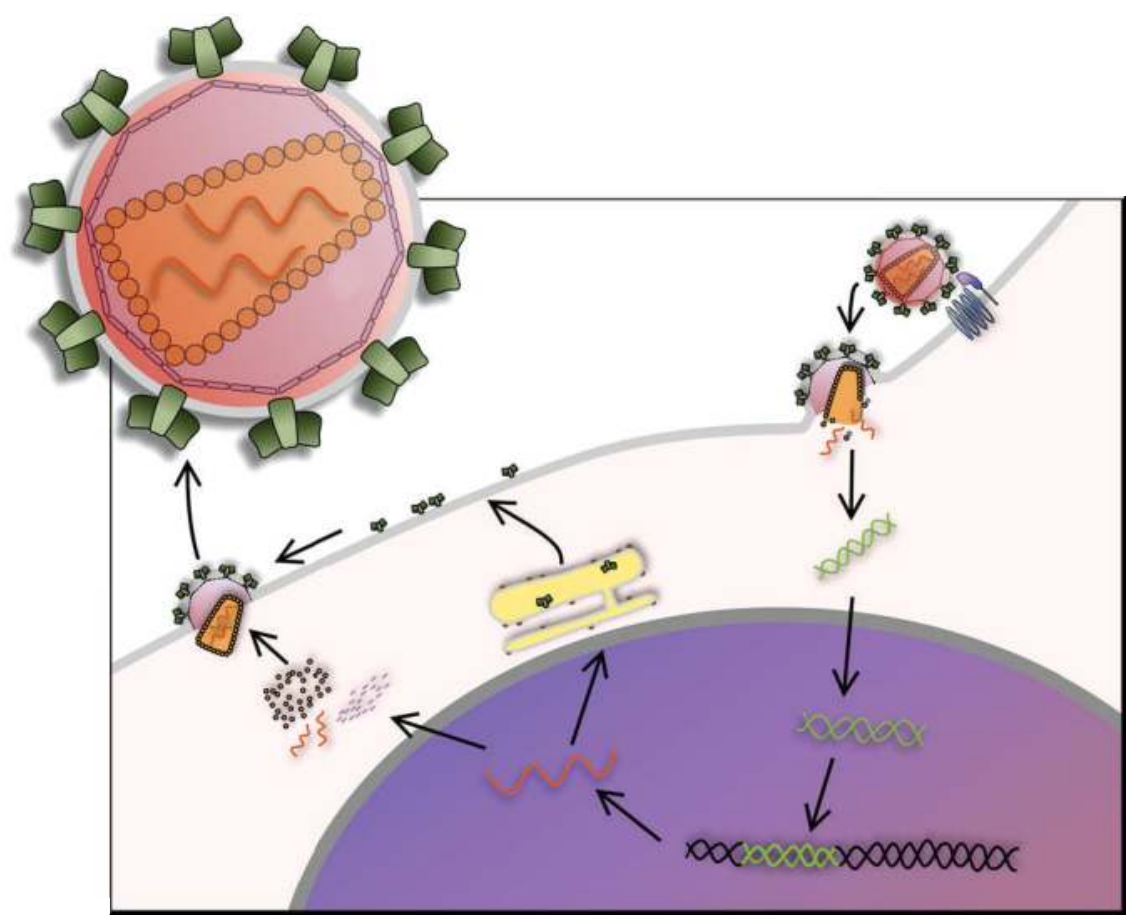

Figure 1. HIV virus is formed by a diploid single strand RNA genome enclosed in a truncated cone capsid with a phospholipidic bilayer envelope, containing the proteins that allow the virus entry into the cells. The HIV-1 infection is mediated by interaction between the proteins of the viral envelope, leukocyte receptor, and coreceptor. This interaction causes the membranes fusion and the uncoating of the virion core. The viral RNA is reverse transcribed in DNA which enters in the nucleus where the integrase enzyme catalyzes the insertion of the viral genome into the genome of the host cell. The expression of integrated viral genome is controlled by the RNA-binding proteins tat and rev. A set of RNAs are transported from the nucleus to the cytoplasm, where they can be translated or packaged. The new core proteins localize near the cell membrane, while the envelope mRNA is translated at the endoplasmic reticulum (ER) and subsequently the envelope proteins are placed on the cell membrane. Finally, the capsid proteins are assembled with the viral genomic RNA, and an immature virion begins to bud from cell surface.

asymptomatic for over 10 years. According to a number of studies, the HLA complex is considered as the most noteworthy genetic marker that is closely related to AIDS progression and highlights the differences in genetic susceptibility of HIV-infected individuals.

\section{HLA polymorphism and genetic predisposition to HIV}

1. Epidemiological survey: Worldwide epidemiological surveys indicate that there is a close relationship between HIV/AIDS and HLA. Although global research reports at different times have demonstrated the involvement of HLA in the differential susceptibility to HIV, the individual HLA locus has not been validated to correlate with HIV infection or AIDS progression. For example, some reports indicate a correlation between HIV susceptibility 
and HLA-B*35 in ethnic groups of Han. However, findings of research on Caucasian individuals did not agree with this notion. HLA-B*07, found among people of African descent, is considered to correlate with the susceptibility to HIV, but the similar correlation has not been reported among people of other ethnic groups. Research on other ethnic groups indicates that HLA-B* 18 and the HLA-A2 either influence HIV-1 infectivity or act as protective genes that inhibit the incidence of AIDS. The homozygous HLA-G 14-bp nsertion/deletion genotype is associated with higher viral load, lower CD4 cell count, and increased mortality compared with HLA-G 14-bp carriers.

Considering the limitation of the small population evaluated, polymorphisms outside the peptide binding region of the HLA class I molecule can play a key role in HIV progression through interaction with other immune-relevant receptors. Some results identify co-operative effects between HLA Class I alleles in the control of HIV-1 in an extended Southern African cohort, and underline complementarity and breadth of the CD8+ T cell targeting as one potential mechanism for this effect. HLA-A-restricted Gag-specific responses can impose selection pressure on HIV. Vaidya et al analyzed the associations between HLA-B alleles and HIV-1 viral replication during acute infection and VLSP in untreated subjects. The results show that the effect of HLA-B*57 on viral control is more pronounced during the later stages of primary HIV-1 infection, while HLA-B*97 is more broadly associated with HIV-1 viral load during primary infection. In HIC (HIV controllers, a unique group of infected individuals who are able to control HIV naturally), HLA-B*57 and the amount of ultrasensitive viral load seem to play a role in the HIV-specific CD8+ T cell responses. Some results provide support for the role of HLA-B*51-restricted CTLs and functional avidity in the control of early HIV-1 infection. HALS (HIV/Highly active antiretroviral therapy-associated lipodystrophy syndrome) is associated with combined low-expression TS (thymidylate synthase) and MTHFR (methylenetetrahydrofolate reductase) associated with high activity polymorphisms but not with HLA$B^{*}$ 40:01 carriage in Caucasian patients with long-term exposure to stavudine. For details, see Table 1.

Richard et al characterized the differential cell surface expression levels of all common HLA$\mathrm{C}$ allotypes and tested directly for effects of HLA-C expression on outcomes of HIV infection in 5243 individuals. They found that HIV peptides presented by higher expressed HLA-C alleles were more likely to elicit CTL responses than peptides presented by low expression HLA-C allotypes, such that higher HLA-C expression was correlated with increased likelihood of cytotoxic T lymphocyte responses and frequency of viral escape mutation. It is also pointed out that HLA-C and HLA-E collaborate to keep the HIV-1 virus at bay. HLA-C can present antigens to CTL, and it is able to inhibit NK cell lysis, but for some reason it is normally expressed on the cell surface at levels approximately 10-fold less than most HLA-A and HLA$\mathrm{B}$ allotypes. The mechanisms that regulate HLA-C expression and the link between this molecule and HIV infection are not yet completely understood. Maybe a new miRNA targeting sequences identified on HLA-C gene and the low level of affinity between $\beta 2 \mathrm{M}$ and the HLA$\mathrm{C}$ heavy chains can explain for this low level of expression. 


\begin{tabular}{|c|c|c|}
\hline \multirow{2}{*}{ Cohorts used in study } & \multicolumn{2}{|l|}{ HLA locus or haplotype or supertype } \\
\hline & Susceptible to HIV or AIDS FP & Non-susceptible to HIV or AIDS SP \\
\hline Han Ethnicity & $B * 35$ & - \\
\hline Zambian & $A^{*} 02-C w^{\star} 16, A^{*} 23-B^{*} 14, A^{*} 23-C w^{\star} 07(F P)$ & $B * 57, B^{*} 39, A^{*} 30-C W^{*} 03(S P)$ \\
\hline European & A1-Cw7-B8-DR3, A24(FP) & - \\
\hline Yi Ethnicity & $B^{\star} 07, B^{\star} 35, B^{\star} 46$ & $B * 55, B^{\star} 44, B^{\star} 78$ \\
\hline Kenya & $A^{*} 29, C w^{*} 07, C w^{*} 08(H T)$ & $B^{*} 18(L T)$ \\
\hline Kenya & $A * 2301$ & A2/6802 supertype(LT) \\
\hline Argentina & $A * 24, B^{*} 18, B^{*} 39$ & $B * 44, B * 55$ \\
\hline African-American & DQB1*0201 & DQB1*0303 \\
\hline Caucasian & $\mathrm{DQB} 1{ }^{*} 0603, \mathrm{DRB} 1{ }^{*} 04$ & $\mathrm{DQB} 1{ }^{*} 0303$ \\
\hline European & $A * 29, B^{\star} 22, D R^{*} 11(F P)$ & $B^{*} 14, C W^{*} 08(S P)$ \\
\hline Caucasian & Heterozygous HLA locus, Type I & $\mathrm{B}^{\star} 35, \mathrm{CW}^{\star} 04$ \\
\hline American & $B \star 54, B * 55, B^{\star} 56$ & - \\
\hline American & - & $B^{\star} 18(L T)$ \\
\hline $\begin{array}{l}\text { Male Caucasian } \\
\text { Homosexuals }\end{array}$ & $B * 35$ & A2 supertype \\
\hline Zimbabwean & HLA-G 14-bp insertion/deletion & \\
\hline European-American & HLA-C low expression & HLA-C high expression \\
\hline
\end{tabular}

FP, fast progression; SP, slow progression; HT, high transmission; LT, low transmission

Table 1. HLA genes, haplotype, and supertype related to HIV-1 susceptibility and AIDS progression

2. In vitro study on HLA and HIV infection susceptibility: It is difficult to extrapolate the common characteristics from the results of epidemiological surveys owing to the abundance of inherent variation. Some researchers try to analyze HLA and HIV infection susceptibility by using in vitro approaches, and speculate the relationship between HLA polymorphism and HIV infection susceptibility or AIDS progression. In vitro studies can better control significant influencing factors such as the type, developmental stage and culturing conditions of cells, type of HIV strain, density of infection, and mechanisms, as well as minimize the variable factors that will have an effect on statistical findings. Second, in vitro experiments facilitate the consistent evaluation of critical factors as well as the application of uniform testing standards. 
Jabri and his colleagues used two strains of HIV-1 and HIV-2 to infect peripheral blood cells with different HLA antigenic specificities, and reported that no single HLA antigen was capable of preventing both types of HIV infection. For example, HLA-B52 showed susceptibility to HIV-1 viral strains, whereas it was not susceptible to HIV-2 viral strains. HLA-B58 showed susceptibility to HIV-2 viral strains, but it was not susceptible to HIV-1 viral strains. HLA-B44 influenced the immune function only against certain HIV-1/2 strains. It is still therefore still ambiguous whether different HLA phenotypes influence the susceptibility to and in vivo effects of HIV infection, which needs to be verified by experiments with larger sample sizes.

HLA-G variant expression has a considerable impact on the control of HIV replication, an effect that seems to be mediated primarily by the protein specificity of CD4(+) T cell responses to HIV Gag and Nef. Numerous studies have been conducted, aimed at observing the expression of the molecule HLA-G in the early stage of infection by HIV and its progression. In 2004, Derrien and colleagues demonstrated that during HIV-1 infection the HLA-G1 isoform was down regulated by a Vpu dependent mechanism, which recognizes a double lysine residues in 4 and 5 positions of the $C$ terminus. The HLA-G1 isoform has the major ability to present viral peptides to CD8+ T lymphocytes; therefore, the recognition of HIV-1 infected cells by CD8+ T lymphocytes could depend on the expression of HLA-G1.More results show that HLAG Treg plays an important role for balancing by stander immune activation and anti-viral immune activity in HIV-1 infection and suggest that the loss of these cells during advanced HIV-1 infection may contribute to immune dysregulation and HIV-1 disease progression. The connection between miRNA, HLA-G expression, and HIV-1 also needs to be further explored because it can reveal novel information about HIV-1 control of the immune system.

3. Statistical analysis of the correlation between HLA gene polymorphism and HIV infection or AIDS susceptibility: Previously published research findings have been analyzed to evaluate the diversity in the correlation of HLA with HIV/AIDS susceptibility. Metaanalysis was anticipated to identify the common features. Research articles that satisfy the selection criteria were retrieved and the original data in the documents were processed and analyzed, wherein B35, B62, DR5, and DR11 were identified as genes that facilitate HIV-1 susceptibility and/or occurrence of AIDS, while A10, B18, B27, B5, and DR1 were identified as protective genes against HIV-1 infection and/or occurrence of AIDS. Since meta-analysis is an observational study, variations are likely to be introduced in each step of the analysis, and the results need to be validated by testing in laboratories with a large sample size.

\section{Possible mechanisms that HLA influence HIV infection susceptibility or AIDS progression}

1. Infection receptors: HIV combines with the CD4 molecule and other coreceptors to invade $\mathrm{T}$ cells during infection. HIV susceptibility is affected when the above-mentioned receptors have natural defects or mutations. So far, it has been verified that receptors of 
chemotactic factors (CCR35 and CXCR4) are coreceptors for HIV-1. When HIV-1 infects target cells, the viruses that combine with CD4 molecules combine with coreceptors in order to enter the target cells. In high-risk populations with close contact to infected individuals but no infection, approximately one-third of the CCR5 alleles undergo deletion mutation; for instance, deletion of 32 bases of CCR5 genes (referred to as CCR5 $\triangle 32$ mutation), which results in a natural immunity of the organism against HIV-1 infection at the gene level. The mutation and polymorphism changes of alleles not only influence the ability of HIV-1 to infect the target cells, but also change the onset and progression of AIDS. It is possible that HLA participates in HIV invading process through similar means, and affects AIDS progression. However, there are no validated reports on HLA's direct participation in HIV-1 infection by acting as the receptor, so far. Therefore, the susceptibility theory, which suggests that HLA acts as a receptor, needs to be subjected to further scientific scrutiny.

2. HLA molecules selection pressure: The antigen-binding groove of HLA molecules combine with a specific epitope of the HIV protein (For example, gap 120), inducing the generation of helper T cells and cytotoxic T cells. In particular, CD8+ cytotoxic T lymphocytes can kill infected CD4 ${ }^{+} \mathrm{T}$ lymphocyte in a direct manner, which can influence virus replication, transmission, and AIDS progression. HLA molecules can employ different epitopes for the same molecule, and stimulate generation of CTLs featuring different quantities as well as specific properties, which has been confirmed in previous studies. Therefore, this can better explain the differences in AIDS progress when individuals with certain HLA phenotypes infected with HIV.

During viral infection, some viruses undergo degradation by cellular proteasome complex, and the cytosolic antigenic peptides are carried into the ER. In this organelle, the peptides are captured by HLA I molecules and then exposed on the cell surface, triggering the cytotoxic activity of the circulating CD8+ T lymphocytes, as described previously. The HIV has devised different ways to evade the immune response including a Nef-dependent mechanism that downregulates the HLA I expression, thus avoiding the recognition of the infected cells by CD8+ T lymphocytes (Fig2). Selectively, Nef alters the expression of HLA-A and -B by recognition of a sequence (Y320SQAASS) present on the cytoplasmic tail of these HLA molecules accelerating their endocytosis from the plasma membrane and blocking the transport of newly synthesized MHC class I molecules to the cell surface. Nef maintains the expression of HLA-C-G and -E unchanged, in order to inhibit the innate response of the natural killer cells (NK). Furthermore, the gp41 protein of the viral envelope upregulates the synthesis of IL-10 by monocytes; in turn, as mentioned before, this cytokine increases the expression of HLA-G molecules to control immune response and facilitate infection.

Meanwhile, under the influence of CTLs that are induced by HLA, mutation of HIV can occur. For example, TW10 peptide fragment (TAILQEQIAW) of gap protein in the HIV-1 virus, has a specific mutation on the residue 242 in the individuals with HLA-B57 or B58 and can revert to the wild type after the viruses detach from HLA-B57 or B58 molecules. This HIV mutation can cause functional loss of specific CTL, and enable HIV to adapt to the HLA environment 


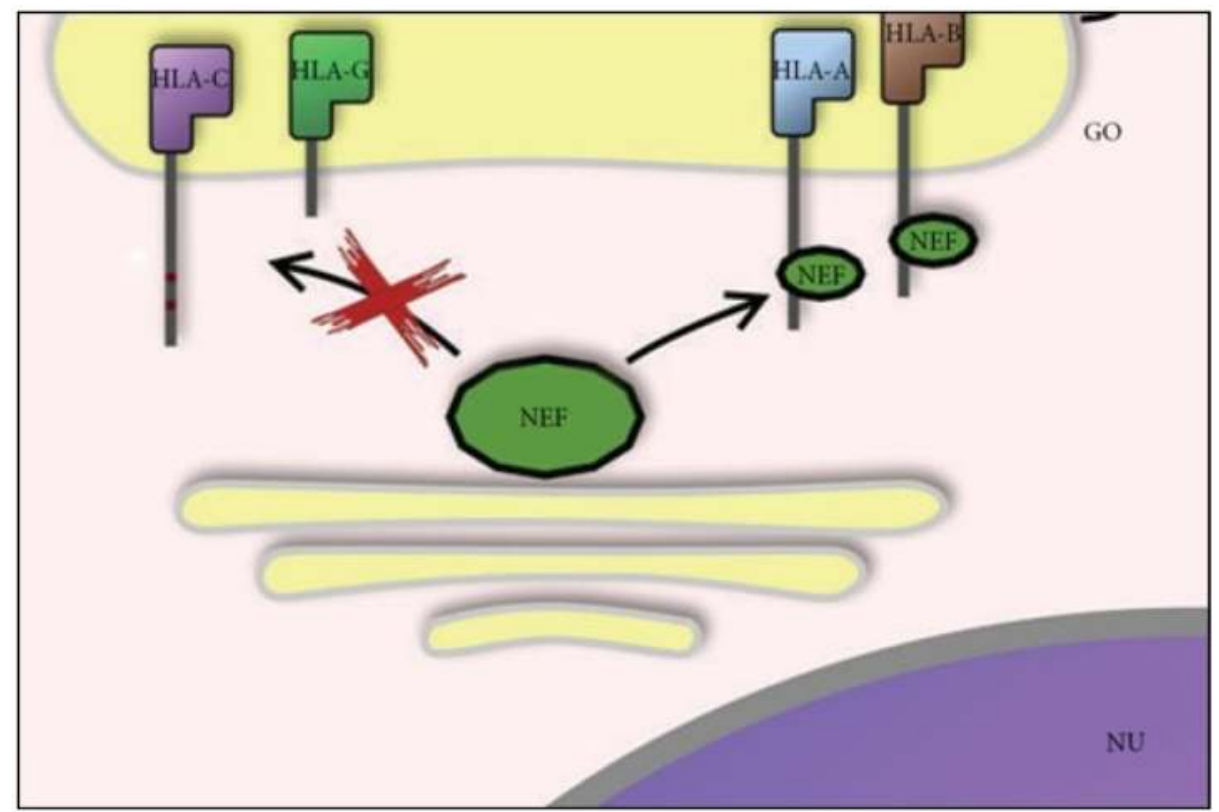

Figure 2. The HIV has devised different ways to evade the immune response, including a Nef-dependent mechanism that downregulates the HLA I expression, thus avoiding the recognition of the infected cells by CD8+ T lymphocytes. Selectively, Nef alters the expression of HLA-A and -B by recognition of a sequence (Y320SQAASS) present on the cytoplasmic tail of these HLA molecules accelerating their endocytosis from the plasma membrane and blocking the transport of newly synthesized MHC class I molecules to the cell surface. Nef maintains the expression of HLA-C, -G, and -E unchanged, in order to inhibit the innate response of the natural killer cells (NK). NU: nucleus; GO: golgi apparatu

and overcome the immune responses, resulting in HIV susceptibility of specific HLA types as well as a rapid progression of AIDS. Children of mothers with AIDS who have similar HLA genes are at a higher risk of being infected with HIV, and they tend to progress to AIDS in a shorter time. The possible reason for this is that the HIV of the mother might have adapted to the HLA environment in the host, and the virus replication increases when HLA similarity is high between the mother and child, which in turn makes it is easier for HIV to propagate and lead to a faster progression of AIDS. But sometimes specific mutation of HIV can also lead to a decrease in its virulence, enabling a reduced susceptibility of individuals towards HIV and a slower progression of AIDS.

\section{Acknowledgements}

Supported by grants from the State Key Development Program for Basic Research of China (No.2003CB515509 and 2009CB522401) and from National Natural Scientific Foundation of China(No.81070450 and 30470751) to Dr. X.-Y.Z. 


\section{Author details}

Fang Yuan and Yongzhi Xi*

*Address all correspondence to: xiyz@yahoo.com

Department of Immunology and National Center for Biomedicine Analysis, Beijing Hospital Affiliated to Academy of Medical Sciences, Beijing, PRC

\section{References}

[1] Grimwood J. Schmutz J. Genomics: six is seventh. Nature, 2003, 425:775-776.

[2] Lefrere JJ, Mariotti M, Morand-Joubert L, et al. Plasma human immunodeficiency virus RNA below 40 Copies $/ \mathrm{ml}$ is rare in untreated persons even in the first years of infection. J Infect Dis, 1999, 180: 526-529.

[3] Tang J, Tang S, Lobashevsky E, et al. Favorable and unfavorable HLA class I alleles and haplotypes in Zambians predominantly infected with clade $\mathrm{C}$ human immunodeficiency virus type 1. J Virol, 2002, 76: 8276-8284.

[4] Xu MY, Hong KX, Deng XL, et al. Association of HLA-B alleles with human immunodeficiency virus type 1 infection in the Yi ethnic group in Sichuan province. Biomed Environ Sci, 2004, 17: 203-208.

[5] MacDonald KS, Matukas L, Embree JE, et al. Human leucocyte antigen supertypes and immune susceptibility to HIV-1, implications for vaccine design. Immunol Lett, 2001, 79: 151-157.

[6] Roe DL, Lewis RE, Cruse JM. Association of HLA-DQ and -DR alleles with protection from or infection with HIV-1. Exp Mol Pathol, 2000, 68: 21-28.

[7] Carrington M, Nelson GW, Martin MP, et al. HLA and HIV-1: heterozygote advantage and $\mathrm{B}^{*} 35-\mathrm{Cw}^{*} 04$ disadvantage. Science, 1999, 283: 1748-1752.

[8] Dorak MT, Tang J, Tang S, et al. Influence of human leukocyte antigen-B22 alleles on the course of human immunodeficiency virus type 1 infection in 3 cohorts of white men. J Infect Dis, 2003, 188: 856-863.

[9] Farquhar C, Rowland-Jones S, Mbori-Ngacha D, et al. Human leukocyte antigen (HLA) B*18 and protection against mother-to-child HIV type 1 transmission. AIDS Res Hum Retroviruses, 2004, 20: 692-697.

[10] Liu C, Carrington M, Kaslow RA, et al. Association of polymorphisms in human leukocyte antigen class I and transporter associated with antigen processing genes with 
resistance to human immunodeficiency virus type 1 infection. J Infect Dis, 2003, 187: 1404-1410.

[11] Al Jabri AA. HLA and in vitro susceptibility to HIV infection. Mol Immunol, 2002, 38: 959-967.

[12] Liu H, Hwangbo Y, Hotle S, et al. Analysis of genetic polymorphisms in CCR5, CCR2, stromal cell-derived factor-1, RANTES, and dendritic cell-specific intercellular adhesion molecule-3-grabbing nonintegrin in seronegative individuals repeatedly exposed to HIV-1. J Infect Dis, 2004, 190: 1055-1058.

[13] Jansen CA, Kostense S, Vandenberghe K, et al. High responsiveness of HLA-B57-restricted Gag-specific CD8+ T cells in vitro may contribute to the protective effect of HLA-B57 in HIV-infection. Eur J Immunol, 2005, 35: 150-158.

[14] Walker BD, Korber BT. Immune control of HIV: the obstacles of HLA and viral diversity. Nat Immunol, 2001, 2: 437-475.

[15] Leslie AJ, Pfafferott KJ, Chetty P, et al. HIV evolution: CTL escape mutation and reversion after transmission. Nat Med, 2004, 10: 282-289.

[16] Kuhn L, Abrams EJ, Palumbo P, et al. Maternal versus paternal inheritance of HLA class I alleles among HIV-infected children: consequences for clinical disease progression. AIDS, 2004, 18: 1281-1289.

[17] Grifoni A, Montesano C, Palma P. et al. Role of HLA-B $\alpha-3$ domain amino acid position 194 in HIV disease progression. Mol Immunol. 2013, 53(4):410-3.

[18] Matthews PC, Listgarten J, Carlson JM, et al. Co-operative additive effects between HLA alleles in control of HIV-1. PLoS One. 2012, 7(10):e47799.

[19] Kloverpris HN, Stryhn A, Harndahl M, et al. HLA-A*68:02-restricted Gag-specific cytotoxic T lymphocyte responses can drive selection pressure on HIV but are subdominant and ineffective. AIDS. 2013, 27(11):1717-23.

[20] Vaidya SA, Streeck H, Beckwith N, et al. Temporal effect of HLA-B*57 on viral control during primary HIV-1 infection. Retrovirology. 2013, 10(1):139.

[21] Lecuroux C, Saez A, Girault I, et al. Both HLA-B*57 and plasma HIV RNA levels contribute to the HIV-specific CD8+ T cell response in HIV controllers. J Virol. 2013.

[22] Yager N, Robinson N, Brown H, et al. Longitudinal analysis of an HLA-B*51-restricted epitope in integrase reveals immune escape in early HIV-1 infection. AIDS. 2013, 27(3):313-23.

[23] Ranasinghe S, Cutler S, Davis I, et al. Association of HLA-DRB1-restricted CD4 T cell responses with HIV immune control. Nat Med. 2013, 19(7):930-3. 
[24] Levitz L, Koita OA, Sangare K, et al. Conservation of HIV-1 T cell epitopes across time and clades: validation of immunogenic HLA-A2 epitopes selected for the GAIA HIV vaccine. Vaccine. 2012, 30(52):7547-60.

[25] Li C, Toth I, Schulze ZW, et al. Functional characterization of HLA-G regulatory T cells in HIV-1 infection. PLoS Pathog. 2013, 9(1):e1003140.

[26] Lenfant F, Pizzato N, Liang S, C. et al. Induction of HLA-G-restricted human cytomegalovirus pp65 (UL83)-specific cytotoxic T lymphocytes in HLA-G transgenic mice. the Journal of General Virology, 2003, 84(2):307-317.

[27] Apps R, Qi Y, Carlson JM, et al. Influence of HLA-C expression level on HIV control. Science. 2013, 340(6128):87-91.

[28] Buranapraditkun S, Hempel U, Pitakpolrat P, et al. A novel immunodominant CD8+ $\mathrm{T}$ cell response restricted by a common HLA-C allele targets a conserved region of Gag HIV-1 BioMed Research International 11 clade CRF01AE infected thais. PLoS ONE. 2011, 6(8):23603.

[29] Fellay J, Shianna KV, .Ge D, et al. A whole-genome association study of major determinants for host control of HIV-1. Science, 2007, 317(5840):944-947.

[30] Trachtenberg E, Bhattacharya T, Ladner M, et al. The HLA-B/-C haplotype block contains major determinants for host control of HIV. Genes and Immunity, 2009, 10(8): 673-677.

[31] Segat L, Catamo E, Fabris A, et al. HLA-G*0105N allele is associated with augmented risk for HIV infection in white female patients. AIDS, 2010, 24(12):1961-1964.

[32] Larsen MH, Zinyama R, Kallestrup P, et al. HLA-G 3' untranslated region 14-base pair deletion: association with poor survival in an HIV-1-infected zimbabwean population. The Journal of Infectious Diseases, 2013, 207(6): 903-906.

[33] Kulkarni S, Savan R, Qi Y, et al, Differential microRNA regulation of HLA-C expression and its association with HIV control. Nature, 2011, 472(7344):495-498.

[34] Gall SL, Buseyne F, Trocha A, Walke BD, et al. Distinct trafficking pathways mediate Nef induced and clathrin-dependent major histocompatibility complex class I downregulation. Journal of Virology, 2000 74(19): 9256-9266.

[35] Kasper MR and Collins KL. Nef-mediated disruption of HLA-A2 transport to the cell surface in T cells. Journal of Virology, 2003 77(5):3041-3049.

[36] Pizzato N, Derrien M, and Lenfant F. The short cytoplasmic tail of HLA-G determines its resistance to HIV-1 Nef-mediated cell surface down regulation. Human Immunology, 2004, 65(11):1389-1396.

[37] Nattermann J, Nischalke HD, Hofmeister V et al, HIV-1 infection leads to increased HLA-E expression resulting in impaired function of natural killer cells. Antiviral Therapy, 2005, 10(1):95-107. 